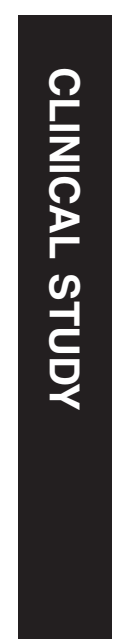

\title{
Impact of socioeconomic status on vision-related quality of life in primary open-angle glaucoma
}

'Department of Ophthalmology, Taipei Veterans General Hospital, Taipei, Taiwan

${ }^{2}$ Faculty of Medicine, National Yang-Ming University School of Medicine, Taipei, Taiwan

${ }^{3}$ Neurological Institute, Taipei Veterans General Hospital, Taipei, Taiwan

${ }^{4}$ Institute of Clinical Medicine, National YangMing University School of Medicine, Taipei, Taiwan

Correspondence: Y-C Ko, Department of Ophthalmology, Taipei Veterans General Hospital, No. 201, Section 2, Shipai Road, Beitou District, Taipei City, Taipei 11217, Taiwan Tel: +88622875 7325; Fax: +886 228757133 . E-mail: koyc@vghtpe.gov.tw

Received: 23 September 2016

Accepted in revised form: 19 April 2017

Published online: 2 June 2017

\begin{abstract}
Purpose To investigate the impact of socioeconomic status (SES) on vision-related quality of life (VRQOL) in patients with primary open-angle glaucoma (POAG). Patients and methods This prospective crosssectional study included consecutive patients with POAG at a tertiary hospital between March 2012 and January 2013. All patients had visual acuity no worse than $20 / 60$ in the better eye and reliable visual field tests. VRQOL was assessed by the validated Taiwan version 25-item National Eye Institute Visual Functioning Questionnaire (NEI VFQ-25). Sociodemographic characteristics, medical history, and ocular parameters were recorded. SES was evaluated based on educational attainment and monthly income, both stratified into three levels. Analysis of variance and linear regression analysis were used to evaluate the relationship between SES, VRQOL, and clinical parameters. Results Among the 186 patients recruited, intergroup differences were not observed among educational or monthly income levels for binocular vision or integrated visual field defects. Patients of lower educational and monthly income levels had lower self-reported general health ratings. After adjustment for visual function, treatment complexity, and general health in the multiple linear regression model, patients with a college degree or higher reported better NEI VFQ-25 scores for the composite score $(P=0.041)$, mental health $(P=0.035)$, and peripheral vision $(P=0.05)$ than did those with education below junior high school. Monthly income levels did not affect the NEI VFQ-25 scores.

Conclusion Educational attainment significantly affects VRQOL in patients with POAG. Additional counseling may be
\end{abstract}

Y-S Kuo ${ }^{1}$, CJ-L Liu ${ }^{1,2}, \mathrm{H}-\mathrm{C}$ Cheng ${ }^{1}$, M-J Chen ${ }^{1,2}$, W-T Chen ${ }^{2,3}$ and $\mathrm{Y}-\mathrm{C} \mathrm{Ko} \mathrm{Ko}^{1,2,4}$ provided to patients with lower educational background to help them cope with the disease.

Eye (2017) 31, 1480-1487; doi:10.1038/eye.2017.99; published online 2 June 2017

\section{Introduction}

Glaucoma is a leading cause of irreversible blindness. ${ }^{1}$ The goal of glaucoma treatment is to maintain visual function, as well as quality of life, in affected patients.

Self-reported vision-related quality of life (VRQOL) allows us to assess the effect of disease and treatments from the patient's perspective, focusing on an individual's subjective satisfaction and functional ability. The impact of glaucoma on VRQOL has been increasingly discussed over the past decades. When compared with patients without glaucoma, several studies have shown that glaucoma patients scored significantly lower on various VRQOL instruments. ${ }^{2-4}$ Previous studies mostly focused on the impact of visual function deficits on VRQOL in patients with glaucoma, indicating that more advanced and faster progression of visual field (VF) defects were associated with lower scores on VRQOL instruments. ${ }^{2,5,6}$ It was also noted that certain locations of the VF defect have different impact on a patient's VRQOL. ${ }^{7,8}$

Socioeconomic status (SES) affects the diagnosis and management of glaucoma from several aspects. Studies from the United Kingdom and Canada found that low SES is a risk factor for advanced glaucoma at presentation. ${ }^{9-11}$ Patients with low educational levels have less glaucoma awareness and poor medication adherence. ${ }^{12}$ Therefore, patients of low SES may require more social support to 
understand the disease process and to adhere to glaucoma treatment that may affect the patient's VRQOL. For glaucoma patients with similar degrees of visual dysfunction, SES may have varying impact on their VRQOL because of differing demands of daily visiondependent tasks. Despite that SES is considered as a confounding factor in several studies dealing with VRQOL in glaucoma patients, few have investigated the impact of SES itself on VRQOL after adjusting for other influential factors. ${ }^{3,4,6}$

The present study aims to understand whether SES has an independent effect on VRQOL in glaucoma patients. A thorough understanding of the effect of SES on VRQOL in glaucoma patients will be helpful in policy decisions to allocate limited medical resources to optimize patients' quality of life.

\section{Materials and methods}

\section{Patients and methods}

From 1 March 2012 to 1 January 2013, consecutive patients with primary open-angle glaucoma (POAG) visiting Taipei Veterans General Hospital were invited to participate in this prospective cross-sectional study that was approved by the institutional review board of the Taipei Veterans General Hospital. Written informed consent was obtained from each participant before enrollment.

Sociodemographic characteristics, medical history, surgical history, and current ophthalmological conditions, including glaucoma medication and dosage frequency, were documented for each patient. A comprehensive ophthalmologic evaluation was performed. Best-corrected visual acuity (VA) of each eye and binocular habitual VA were recorded using the Snellen E chart. Binocular habitual VA was converted to logMAR value for further analysis. Automated perimetry was performed for each eye separately using the 24-2 Swedish interactive threshold algorithm standard of the Humphrey Field Analyzer 750i (version 4.2, Zeiss-Humphrey Instruments, Dublin, CA, USA), and were subsequently combined and converted to integrated VF (IVF) using the best sensitivity method. ${ }^{13,14}$ The better raw sensitivity (decibels) and less negative total deviation from each pair of the spatially corresponding points of the two eyes were chosen to represent the sensitivity and total deviation of the particular locus in the IVF, excluding the two most nasal points. The mean deviation of the IVF (IVF-MD) was the mean of the total deviation values of each test point. 8,14

POAG was diagnosed by glaucoma specialists (CJ-LL, $\mathrm{Y}-\mathrm{CK}$, and $\mathrm{M}-\mathrm{JC}$ ) in the presence of glaucomatous optic disc changes with corresponding retinal nerve fiber layer loss and reproducible VF defects, and a normal open angle on gonioscopy. Glaucomatous optic disc changes were defined as an enlarged optic disc cupping along with generalized neuroretinal rim thinning or focal notching. Glaucomatous VF defects were defined as those with a cluster of $\geq 3$ nonedge contiguous points in the same hemifield with probabilities of $<5 \%$ on the pattern deviation map, including at least one point with a probability of $<1 \%$. In addition, the VF tests were classified as outside normal limit in glaucoma hemifield tests or had a pattern SD outside $95 \%$ of normal limits. Patients who satisfied the following criteria were enrolled: (1) best-corrected VA no worse than 20/60 in the better eye, (2) intraocular pressure controlled at or below $25 \mathrm{~mm} \mathrm{Hg}$ and (3) reliable VF results performed within 3 months with $<30 \%$ fixation losses, $15 \%$ false positive responses, and $15 \%$ false negative responses. Patients who had a patent peripheral iridotomy, laser or incisional glaucoma surgery within 3 months, histories of refractive surgery, and other ocular or neurological diseases that could result in VF defect were excluded. Patients with severe comorbidities known to affect quality of life, such as dementia, end-stage renal disease, dysfunctional parkinsonism, or systemic malignancy, were also excluded.

\section{SES stratification}

SES was evaluated based on the self-reported educational attainment and monthly income data, each stratified into three levels. Educational levels were categorized into low (up to junior high or ninth grade, the compulsory education level in Taiwan since 1968), middle (high school or other secondary education), and high (college or above). Monthly income was stratified into low $(<30000$ New Taiwan Dollars (NTD)), middle (30 000-60 000 NTD), and high (>60000 NTD). The stratification was based on the national income per capita in 2012 (44 739 NTD) and that of the retired population aged $\geq 65$ years (32 875 NTD) in Taiwan.

\section{VRQOL assessment}

The validated Taiwan version of the 25-item National Eye Institute Visual Functioning Questionnaire (NEI VFQ-25) was used in this study for assessment of VRQOL. ${ }^{15} \mathrm{NEI}$ VFQ-25 is a widely used questionnaire designed to assess the perception of visual function across a range of daily activities and quality of life in patients with chronic eye diseases. It consists of 25 vision-targeted questions representing 11 vision-related subscales plus an additional single item of a 5-level general health rating question. ${ }^{16}$ The score of each subscale ranges from 0 to 100, with higher scores indicating better VRQOL. The NEI VFQ-25 composite score is calculated by averaging the subscale scores, excluding the general health rating question, thus representing the overall result for VRQOL. All participants 
Table 1 Demographic data, ocular characteristics, and NEI-VFQ-25 scores of different educational groups

\begin{tabular}{|c|c|c|c|c|c|}
\hline & Junior high or below & Senior high & College or above & Total & $\begin{array}{c}\text { Intergroup difference } \\
(\mathrm{P}-\mathrm{value})^{\mathrm{a}}\end{array}$ \\
\hline General & \multicolumn{5}{|c|}{ Number $(\%)$ or mean $(S D)$} \\
\hline Number of cases & $29(15.59)$ & $58(31.18)$ & $99(53.23)$ & $186(100.00)$ & \\
\hline Age (years) & $68.62(10.15)$ & $59.98(12.40)$ & $55.81(12.84)$ & $59.11(13.02)$ & $<0.001^{\mathrm{b}}$ \\
\hline Gender & & & & & $<0.001^{c}$ \\
\hline Male & $13(44.82)$ & $38(65.52)$ & $80(80.80)$ & $131(70.43)$ & \\
\hline Female & $16(55.17)$ & $20(34.48)$ & 19 (19.19) & $55(29.57)$ & \\
\hline Income & & & & & $<0.001^{\mathrm{c}}$ \\
\hline$<30000$ NTD & $25(86.21)$ & $34(58.62)$ & $34(34.34)$ & $93(50)$ & \\
\hline 30 000-60 000 NTD & $2(6.90)$ & $17(29.31)$ & $17(17.17)$ & $36(19.36)$ & \\
\hline$>60000$ NTD & $2(6.90)$ & $7(12.07)$ & $48(48.48)$ & $57(30.65)$ & \\
\hline Employment status & & & & & $<0.001^{\mathrm{c}}$ \\
\hline Employed & $3(10.34)$ & $28(48.28)$ & $62(62.62)$ & $93(50)$ & \\
\hline Retired/unemployed & $26(89.66)$ & $30(51.72)$ & $37(37.37)$ & $93(50)$ & \\
\hline \multicolumn{6}{|l|}{ Ocular characteristics } \\
\hline LogMAR binocular VA & $0.062(0.11)$ & $0.036(0.09)$ & $0.03(0.11)$ & $0.37(0.1)$ & $0.301^{\mathrm{d}}$ \\
\hline Integrated visual fields (MD) & $-6.77(5.57)$ & $-4.93(5.31)$ & $-4.22(4.00)$ & $-4.84(4.76)$ & $0.054^{\mathrm{d}}$ \\
\hline Intraocular pressure $(\mathrm{mm} \mathrm{Hg})$ & $14.88(2.61)$ & $14.90(2.80)$ & $14.86(2.60)$ & $14.88(2.65)$ & $0.933^{\mathrm{d}}$ \\
\hline Number of glaucoma medications & $1.55(0.95)$ & $1.86(0.96)$ & $1.87(0.90)$ & $1.82(0.93)$ & $0.311^{\mathrm{d}}$ \\
\hline Eyedrop frequency (times/day) & $1.79(1.21)$ & $1.97(1.09)$ & $2.1(1.11)$ & $2.01(1.12)$ & $0.441^{\mathrm{d}}$ \\
\hline Previous ocular surgery & $12(41.38)$ & $23(39.66)$ & $40(40.40)$ & 75 (40.32) & $0.988^{c}$ \\
\hline \multicolumn{6}{|l|}{ Medical conditions } \\
\hline Diabetes & $6(20.69)$ & $10(17.24)$ & $6(6.06)$ & $22(11.83)$ & $0.031^{\mathrm{d}}$ \\
\hline Hypertension & $16(55.17)$ & $18(31.03)$ & $21(21.21)$ & $55(29.57)$ & $0.002^{\mathrm{d}}$ \\
\hline Coronary heart disease & $3(10.34)$ & $0(0.00)$ & $3(3.03)$ & $6(10.34)$ & $0.037^{\mathrm{d}}$ \\
\hline Insomnia & $4(13.79)$ & $3(5.17)$ & $2(2.02)$ & $9(4.84)$ & $0.034^{\mathrm{d}}$ \\
\hline \multicolumn{6}{|l|}{ NEI VFQ-25 scores } \\
\hline General health & $23.28(18.81)$ & $44.40(24.80)$ & $46.21(24.84)$ & $42.07(25.22)$ & $<0.001^{\mathrm{d}}$ \\
\hline General vision & $43.10(22.06)$ & $43.10(19.19)$ & 47.47 (19.72) & $45.43(19.95)$ & $0.184^{\mathrm{d}}$ \\
\hline Ocular pain & $75.00(24.09)$ & $77.8(20.14)$ & $78.5(19.72)$ & $77.76(20.51)$ & $0.875^{\mathrm{d}}$ \\
\hline Near activities & $70.00(30.71)$ & $74.40(26.22)$ & $82.4(21.88)$ & $78.10(25.03)$ & $0.067^{\mathrm{d}}$ \\
\hline Distance activities & $86.59(16.66)$ & $86.55(20.08)$ & 86.47 (17.78) & $86.51(18.33)$ & $0.820^{\mathrm{d}}$ \\
\hline Social functioning & $93.00(12.54)$ & 94.68 (13.65) & $93.88(12.95)$ & $94.00(13.05)$ & $0.457^{\mathrm{d}}$ \\
\hline Mental health & $63.79(28.11)$ & $75.10(20.59)$ & 76.07 (18.85) & $73.86(21.39)$ & $0.146^{\mathrm{d}}$ \\
\hline Role difficulties & $65.08(31.04)$ & 74.35 (26.53) & 75.75 (27.48) & $73.66(27.87)$ & $0.202^{\mathrm{d}}$ \\
\hline Dependency & $83.04(22.21)$ & 91.09 (17.23) & $92.25(14.73)$ & $90.45(17.07)$ & $0.052^{\mathrm{d}}$ \\
\hline Driving & $75.52(15.66)$ & $72.31(30.07)$ & 77.19 (21.62) & $75.34(24.20)$ & $0.616^{\mathrm{d}}$ \\
\hline Color vision & $94.74(22.94)$ & 96.43 (12.99) & $97.78(10.36)$ & 96.97 (13.17) & $0.787^{\mathrm{d}}$ \\
\hline Peripheral vision & $84.00(23.80)$ & $92.86(12.50)$ & 92.58 (15.59) & $91.36(16.49)$ & $0.105^{\mathrm{d}}$ \\
\hline Composite score & 74.59 (15.10) & 81.31 (12.03) & $83.00(12.02)$ & $81.16(12.81)$ & $0.013^{\mathrm{d}}$ \\
\hline
\end{tabular}

Abbreviations: NEI VFQ-25, 25-item National Eye Institute Visual Functioning Questionnaire; NTD, New Taiwan Dollars; VA, visual acuity; MD, mean deviation.

Factors reaching statistical significance of $P<0.05$ are shown in bold.

${ }^{\mathrm{a}} P$-values were calculated using the ANOVA, ${ }^{\mathrm{b}} \chi^{2}, \mathrm{c}$ or Kruskal-Wallis test ${ }^{\mathrm{d}}$ depending on the type and normality of data.

completed the questionnaire with the assistance of a single trained interviewer at the time of enrollment.

\section{Statistical analyses}

The sample size was calculated based on an estimated effect size of 0.25 for the composite score from the pilot study with the power being set at 0.8 , and an $\alpha$-error of 0.05 . This required at least 159 participants for this study.
Intergroup differences of the sociodemographic characteristics, clinical parameters, and NEI VFQ-25 scores were evaluated through one way analysis of variance (ANOVA) or the Kruskal-Wallis test for continuous variables based on the normality of data distribution analyzed by the Shapiro-Wilk test; the categorical variables were analyzed by the $\chi^{2}$ test. Missing data were excluded from analyses.

As the NEI VFQ-25 scores were not normally distributed, correlation between the scores and individual 
factors were assessed with the Kruskal-Wallis test, Mann-Whitney U-test, and Spearman's rank correlation (Table 3). Factors correlating with the subscales or the composite score with a $P$-value of $<0.2$ were selected as a covariate in the following multiple linear regression analysis. Multiple linear regression models were built for the composite score and subscales in which education or income were correlated with the scores having a $P$-value of $<0.2$ in the univariate analysis. As our study group predominantly comprised elderly and male participants, age and gender were forced to be part of the multiple linear regression model to adjust for possible confounding effects. A two-sided $P$-value of $<0.05$ was considered statistically significant. All analyses were conducted using SPSS (version 18.0.0, SPSS Inc., Chicago, IL, USA).

\section{Results}

The present study included 186 patients with POAG (131 men and 55 women), with a mean age (SD) of 59.11 (13.02) years. Tables 1 and 2 list the demographic data, ocular parameters, medical comorbidities, and NEI VFQ-25 scores of the patients with different educational and monthly income levels. The nonresponse rates for the NEI VFQ-25 questionnaire were 5.3\% for questions related to near-vision activities, $9.1 \%$ for distant-vision activities, $5.9 \%$ for social functioning, $28.5 \%$ for driving difficulties, $11.3 \%$ for color vision, and $11.3 \%$ for peripheral vision.

The ocular parameters did not differ across the three income or educational levels; however, patients with lower educational attainment tended to have more advanced VF defects. Further analysis revealed that the prevalence of patients with advanced glaucoma, defined by a IVF-MD worse than $-12 \mathrm{~dB}$, was higher in patients with low education levels $(20.69 \%)$ than those with middle $(8.62 \%)$ or high $(4.04 \%)$ education levels $(P=0.015)$. Men were more likely to have high educational and monthly income levels than women. Patients with high educational levels were more likely to have high monthly incomes and vice versa. Most patients $(86.2 \%)$ of the low education group had low monthly income levels. On the other hand, patients with low monthly income levels had more variable educational attainment levels. This may be explained by the fact that almost $50 \%$ of the study patients were retired with limited earnings but varying educational backgrounds. Selfreported general health $(P<0.001)$ and the NEI VFQ-25 composite score $(P=0.013)$ differed among patients with different educational attainment, with higher scores in patients with high education levels. Monthly income levels did not affect the NEI VFQ-25 scores; however, patients with low income levels had lower general health ratings.
Men, better general health ratings, better binocular visual function, less glaucoma medications, lower eyedrop instillation frequency, and higher educational levels were associated with higher NEI VFQ-25 composite scores in the univariate analysis. Multiple linear regression analysis revealed that general health $(P=0.004)$, binocular VA $(P=0.006)$, IVF-MD $(P=0.002)$, and educational attainment (low vs high, $P=0.041$ ) were the independent determinants of the NEI VFQ-25 composite score, after adjustment for age and gender (Table 3). Older patients had higher NEI VFQ-25 composite scores. Multiple linear regression analysis was also performed for the NEI VFQ-25 subscales associated with educational attainment with a $P$-value of $<0.2$ (Table 1), including general vision, near activity, mental health, dependency, and peripheral vision (Supplementary Table 1). Educational attainment (low vs high) was independently associated with mental health $(P=0.035)$ and peripheral vision $(P=0.050)$.

\section{Discussion}

In addition to binocular VA and IVF-MD, this study found that general health and educational attainment are independent determinants of VRQOL, as indicated by the composite score of NEI VFQ-25.

Our findings are in line with previous literature that both SES and general health condition may affect VRQOL. ${ }^{17,18}$ In the Los Angeles Latino Eye Study, sociodemographic characteristics, including age, gender, income, and acculturation, accounted for $6 \%$ of the variations in the NEI VFQ-25 composite score, whereas self-reported comorbidities accounted for $12 \%$ of VRQOL variances in the general population. ${ }^{18}$ However, the aforementioned study did not investigate the interaction between general health and sociodemographic characteristics. In our study, patients with lower educational attainment were more likely to have diabetes $(P=0.031)$, hypertension $(P=0.002)$, coronary heart disease $(P=0.036)$, and insomnia $(P=0.034)$, as well as a lower score for general health. Therefore, the single general health rating question of the NEI VFQ-25 was considered a predictor of the overall health condition in the study participants. Furthermore, POAG patients with lower income and educational levels had poorer selfreported general health ratings; educational levels and self-reported general health are both independent determinants of the NEI VFQ-25 composite score. This finding emphasizes the importance of evaluating the subjective general health condition in our patients by a simple question and providing additional support to those with lower scores.

Glaucoma comprehension improved VRQOL and reduced psychological disturbance in patients. ${ }^{19}$ 
Table 2 Demographic data, ocular characteristics, and NEI-VFQ-25 results of different income groups

\begin{tabular}{|c|c|c|c|c|c|}
\hline & $L o w^{a}$ & Middle $^{\mathrm{a}}$ & $H_{i g h}{ }^{\mathrm{a}}$ & Total & $\begin{array}{c}\text { Intergroup difference } \\
(\mathrm{P}-\mathrm{value})^{\mathrm{b}}\end{array}$ \\
\hline General & \multicolumn{5}{|c|}{ Number $(\%)$ or mean $(S D)$} \\
\hline Number of cases & $93(50.00)$ & $36(19.35)$ & 57 (30.65) & $186(100.00)$ & \\
\hline Age (years) & $65.82(12.74)$ & $52.72(11.30)$ & $52.19(8.22)$ & $59.11(13.02)$ & $<0.001^{\mathrm{c}}$ \\
\hline Gender & & & & & $<0.001^{\mathrm{d}}$ \\
\hline Male & $52(55.91)$ & $26(72.22)$ & 53 (91.14) & $131(70.43)$ & \\
\hline Female & $41(44.09)$ & $10(27.78)$ & $4(7.02)$ & 55 (29.57) & \\
\hline Education & & & & & $<0.001^{\mathrm{d}}$ \\
\hline Junior high or below & $25(26.88)$ & $2(5.56)$ & $2(3.51)$ & $29(15.59)$ & \\
\hline Senior high & $34(36.56)$ & $17(47.22)$ & $7(12.28)$ & $58(31.18)$ & \\
\hline College or above & $34(36.56)$ & $17(47.22)$ & $48(84.21)$ & $99(53.23)$ & \\
\hline Employment Status & & & & & $<0.001^{\mathrm{d}}$ \\
\hline Employed & $9(9.68)$ & $31(86.11)$ & $53(92.98)$ & $93(50.00)$ & \\
\hline Retired/unemployed & $84(90.32)$ & $5(13.88)$ & $4(7.02)$ & $93(50.00)$ & \\
\hline \multicolumn{6}{|l|}{ Ocular characteristics } \\
\hline LogMAR binocular VA & $0.053(0.12)$ & $0.036(0.09)$ & $0.012(0.08)$ & $0.037(0.11)$ & $0.167^{\mathrm{e}}$ \\
\hline Integrated visual fields (MD) & $-5.26(5.30)$ & $-4.28(3.70)$ & $-4.52(4.41)$ & $-4.84(4.76)$ & $0.817^{\mathrm{e}}$ \\
\hline Intraocular pressure $(\mathrm{mm} \mathrm{Hg})$ & $15.14(2.84)$ & $14.94(2.17)$ & $14.40(2.58)$ & $14.88(2.65)$ & $0.323^{\mathrm{e}}$ \\
\hline Number of glaucoma medications & $1.77(0.99)$ & $1.75(0.84)$ & $1.93(0.88)$ & $1.82(0.93)$ & $0.548^{\mathrm{e}}$ \\
\hline Eyedrop frequency (times/day) & $1.96(1.20)$ & $1.89(0.95)$ & $2.18(1.09)$ & $2.01(1.12)$ & $0.380^{\mathrm{e}}$ \\
\hline Previous ocular surgery & $38(40.86)$ & $16(44.44)$ & $21(36.84)$ & $75(40.32)$ & $0.759^{\mathrm{d}}$ \\
\hline \multicolumn{6}{|l|}{ Medical conditions } \\
\hline Diabetes & $15(16.13)$ & $4(11.11)$ & $3(5.26)$ & $22(11.83)$ & $0.135^{\mathrm{e}}$ \\
\hline Hypertension & $44(47.31)$ & $6(16.67)$ & $5(8.77)$ & $55(29.57)$ & $<0.001^{\mathrm{e}}$ \\
\hline Coronary heart disease & $5(5.38)$ & $1(2.78)$ & $0(0.00)$ & $6(3.26)$ & $0.194^{\mathrm{e}}$ \\
\hline Insomnia & $5(5.38)$ & $1(2.78)$ & $3(5.26)$ & $9(4.84)$ & $0.814^{\mathrm{e}}$ \\
\hline \multicolumn{6}{|l|}{ NEI VFQ-25 scores } \\
\hline General health & $36.02(24.83)$ & $41.67(23.91)$ & $52.19(23.76)$ & $42.07(25.22)$ & $<0.001^{\mathrm{e}}$ \\
\hline General vision & $45.97(21.56)$ & $45.14(16.71)$ & $44.74(19.32)$ & $45.43(19.95)$ & $0.991^{\mathrm{e}}$ \\
\hline Ocular pain & $80.24(21.12)$ & $77.43(19.32)$ & $73.90(19.94)$ & $77.76(20.51)$ & $0.089^{\mathrm{e}}$ \\
\hline Near activities & $75.62(27.93)$ & $81.55(21.37)$ & $79.86(21.12)$ & $89.10(25.03)$ & $0.685^{\mathrm{e}}$ \\
\hline Distance activities & 87.35 (19.71) & $88.41(14.15)$ & $84.23(18.46)$ & 86.51 (18.33) & $0.217^{\mathrm{e}}$ \\
\hline Social functioning & 91.96 (16.08) & $96.88(7.54)$ & $95.23(10.06)$ & $86.51(18.34)$ & $0.372^{\mathrm{e}}$ \\
\hline Mental health & $72.65(23.14)$ & $77.60(17.12)$ & $73.46(20.91)$ & 73.86 (21.39) & $0.573^{\mathrm{e}}$ \\
\hline Role difficulties & 73.93 (30.48) & $77.43(18.86)$ & $70.83(28.28)$ & $73.66(27.87)$ & $0.612^{\mathrm{e}}$ \\
\hline Dependency & $90.32(17.94)$ & $91.44(12.68)$ & $90.06(18.26)$ & $90.45(17.08)$ & $0.936^{\mathrm{e}}$ \\
\hline Driving & $73.46(26.59)$ & $70.67(28.30)$ & 79.56 (18.67) & $75.34(24.20)$ & $0.371^{\mathrm{e}}$ \\
\hline Color vision & 95.73 (16.10) & $100(0.00)$ & 97.12 (11.77) & 96.97 (13.18) & $0.309^{\mathrm{e}}$ \\
\hline Peripheral vision & $91.98(14.16)$ & $91.94(13.52)$ & $90.09(21.00)$ & $91.36(16.49)$ & $0.987^{\mathrm{e}}$ \\
\hline Composite score & $80.76(13.52)$ & $83.15(9.46)$ & 80.55 (13.52) & $81.16(12.81)$ & $0.830^{\mathrm{e}}$ \\
\hline
\end{tabular}

Abbreviations: NEI VFQ-25, 25-item National Eye Institute Visual Functioning Questionnaire; SD, standard deviation; VA, visual acuity; MD, mean deviation.

Factors reaching statistical significance of $P<0.05$ are shown in bold.

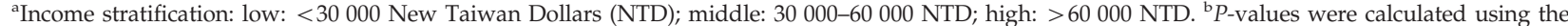
ANOVA, ${ }^{c} \chi^{2},{ }^{d}$ or Kruskal-Wallis test ${ }^{\mathrm{e}}$ depending on the type and normality of data.

Education may be crucial for effective knowledge transfer and finding support to compensate for glaucoma-related visual dysfunctions and psychological distress. Patients with glaucoma having higher educational levels exhibit better utility values and less fear of blindness. ${ }^{20,21}$ After adjustment for visual dysfunction and general health condition, the effect of education on VRQOL was significant when comparing study patients with education below junior high school with those who attended colleges or above in our study. Similarly, a study in China found that the impact of education on VRQOL is significant only in patients with secondary school or higher degrees, compared with those who were illiterate or had primary school educational levels. ${ }^{22}$ However, Lisboa et $a l^{6}$ reported that whether or not the patient had an educational degree of high school or above did not significantly affect VRQOL. Education may have to reach a certain level to have significant impact on improving the 
Table 3 Factors affecting the NEI VFQ-25 composite score in univariate and multiple linear regression models

\begin{tabular}{lrrr}
\hline Factors & $\begin{array}{c}\text { Univariate } \\
\text { analysis }^{\mathrm{a}}\end{array}$ & \multicolumn{2}{c}{$\begin{array}{c}\text { Multiple linear } \\
\text { regression }\end{array}$} \\
\cline { 3 - 5 } & P-value & $\beta$ & P-value \\
\hline Age & $0.621^{\mathrm{c}}$ & 0.16 & $\mathbf{0 . 0 3 4}$ \\
Gender & $0.033^{\mathrm{d}}$ & 0.08 & 0.247 \\
General health & $<0.001^{\mathrm{c}}$ & 0.20 & $\mathbf{0 . 0 0 4}$ \\
Binocular LogMAR VA & $0.004^{\mathrm{c}}$ & -0.20 & $\mathbf{0 . 0 0 6}$ \\
Integrated visual field (MD) & $0.003^{\mathrm{c}}$ & 0.22 & $\mathbf{0 . 0 0 2}$ \\
Number of glaucoma medications & $0.001^{\mathrm{c}}$ & 0.02 & 0.868 \\
Glaucoma medication dosing & $0.001^{\mathrm{c}}$ & -0.24 & 0.09 \\
frequency, (times/day) & & & \\
Education & $0.008^{\mathrm{e}}$ & & \\
Education difference (low-middle) & & 0.15 & 0.141 \\
Education difference (low-high) & & 0.23 & $\mathbf{0 . 0 4 1}$ \\
Previous ocular surgery & $0.311^{\mathrm{c}}$ & & \\
Previous glaucoma surgery & $0.790^{\mathrm{c}}$ & & - \\
Income & $0.998^{\mathrm{e}}$ & & - \\
Adjusted $R^{2}$ & & 0.237 & \\
\hline
\end{tabular}

Abbreviations: NEI VFQ-25, 25-item National Eye Institute Visual Functioning Questionnaire; VA, visual acuity; MD, mean deviation. Factors reaching statistical significance of $P<0.05$ are shown in bold. ${ }^{a}$ Univariate analysis was performed using Kruskal-Wallis test, ${ }^{e}$ Mann-Whitney $U$-test ${ }^{\mathrm{d}}$ or Spearman's rank correlation. ${ }^{\mathrm{c}}$

${ }^{b}$ Factors that reached a $P$-value of $<0.2$ in univariate analysis were included in the multiple regression model. Age and gender were forced to be part of the model.

VRQOL in patients with glaucoma. Different cultural backgrounds may also impact the effect of education on VRQOL. In addition to the educational background of patients, educational lectures aiming to improve the disease understanding may have an immediate effect in improving VRQOL in patients with glaucoma. ${ }^{23}$ Therefore, education for better understanding of glaucoma and its management should be provided to patients, particularly those of lower SES, to improve treatment adherence and their quality of life. However, in study patients with advanced glaucoma, defined as IVFMD $<-12 \mathrm{~dB}$, IVF-MD was the only independent predictor of VRQOL outcome, and education was no longer an influencing factor. Therefore, visual function remains the primary determinant of VRQOL.

In our study, lower monthly income was not an independent predictor of worse VRQOL in patients with POAG. This differs from the reports by Zhou et al, ${ }^{24}$ that evaluated VRQOL of glaucoma patients in China with the Chinese-version Glaucoma Quality of Life -15 questionnaire (GQL-15) and revealed that patients with greater economic burden had poorer GQL-15 scores. The difference may be attributed to the different health-care system and sociodemographic characteristics of participants between the two studies. Health-care utilization is often hindered among socioeconomically deprived patients. However, the National Health
Insurance System in Taiwan, aiming to provide equal access of health care to all citizens, reduces the economic barrier to health-care resources with extensive coverage of medical expenses. Another explanation is that the selfreported monthly income may not have reflected the socioeconomic status of the retired population in our study. Most study patients $(89.2 \%)$ in the low income group were retired and did not have a regular salary, and we did not inquire into the details of the individual's household income, pension, or bank savings. Considering these factors, SES in our study might be more fairly represented by levels of educational attainment instead of monthly income. We used the NEI VFQ-25 to assess VRQOL rather than the glaucoma-specific questionnaires, such as GQL-15, because it comprehensively addresses the issue of VRQOL, and has a validated Taiwan version. ${ }^{8}$

Educational attainment not only affected the composite score, but also the subscales, mental health, and peripheral vision in the multiple linear regression analysis. The questions asked for mental health were focused on the distress, frustration, embarrassment, and loss of control caused by visual impairment. ${ }^{16}$ Therefore, we may assume that patients with higher education have better comprehension of glaucoma that may help them cope with disease-related dysfunction and psychological distress. ${ }^{19}$ However, the presence of questionnaire response bias is possible, such as social desirability, with which high-SES participants tend to have more positive responses. The association of higher scores for peripheral vision with higher education status deserves future exploration. It may be attributed to the fact that there were significantly more patients with advanced glaucoma in the low education group, and thus a higher possibility of peripheral visual field impairment. However, we could not exclude the possibility that this finding resulted from disparate locations of visual field defects across different education groups, as the location of the visual field defects were not included for comparison. Another possible explanation is that patients with higher education are more aware of the fact that glaucoma causes peripheral visual defects, possibly paying more attention to their surroundings in daily life and adapting better to their visual impairment. To our knowledge, this study is one of the first to investigate the impact of education on specific vision-related subscales of VRQOL.

The present study has several limitations. Our sample size was relatively small and comprised only Taiwanese participants. We had excluded those with corrected VA of the better eye worse than 20/60, and therefore may have underestimated the impact of advanced disease on VRQOL that is more prevalent in patients with low SES. Furthermore, our findings cannot be generalized to other types of glaucoma, such as primary angle-closure glaucoma, because SES has variable effects on the 
susceptibility and diagnoses of angle-closure glaucoma and POAG. ${ }^{25}$ Compared with people in the United States, Japan, and Korea, those in Taiwan have a younger average retirement age of 62 years. ${ }^{26}$ Many patients with high educational attainment were classified into the low monthly income group because of retirement. Therefore, monthly income may not have appropriately represented the SES of our patients. Future studies may consider using the health insurance premium or self-reported household income for improved assessment of an individual's economical status, particularly in the retired population.

\section{Conclusion}

Educational attainment, an index of SES, is an independent determinant of VRQOL in glaucoma patients, in addition to glaucoma-related visual dysfunction. Educational programs for increasing glaucoma awareness and knowledge may be a key to improving quality of life in patients with glaucoma, especially in socioeconomically deprived patients, in addition to control of the disease. More information and counseling should be given in clinical settings to those of lower educational background and poorer general health ratings to help them cope with glaucoma and the associated distress.

\section{Summary}

What was known before
Impaired visual function, including best-corrected visual
acuity and visual field defects, affects vision-related
quality of life in patients with glaucoma.
What this study adds
Educational attainment is an independent determinant of
vision-related quality of life in patients with primary open-
angle glaucoma. Certain subscales of vision-related quality
of life, such as peripheral vision and mental health, may
also be affected by education. Providing knowledge and
counseling to patients with glaucoma of lower educational
background may be beneficial.

\section{Conflict of interest}

The authors declare no conflict of interest.

\section{Acknowledgements}

We thank Ms Hsin-Yi Huang for her knowledge and technical support in statistical analysis. The conduction of this study was supported by Taipei Veterans General Hospital, Taipei, Taiwan (Grant nos. V102C-186 and V103C-192).

\section{References}

1 Quigley HA, Broman AT. The number of people with glaucoma worldwide in 2010 and 2020. Br J Ophthalmol 2006; 90: 262-267.

2 Gutierrez P, Wilson MR, Johnson C, Gordon M, Cioffi GA, Ritch $\mathrm{R}$ et al. Influence of glaucomatous visual field loss on health-related quality of life. Arch Ophthalmol 1997; 115: 777-784.

3 Sherwood MB, Garcia-Siekavizza A, Meltzer MI, Hebert A, Burns AF, McGorray S. Glaucoma's impact on quality of life and its relation to clinical indicators. Ophthalmology 1998; 105: $561-566$.

4 Freeman EE, Muñoz B, West SK, Jampel HD, Friedman DS. Glaucoma and the quality of life- the Salisbury eye evaluation. Ophthalmology 2008; 115: 233-238.

5 McKean-Cowdin R, Wang Y, Wu J, Azen SP, Varma R. Impact of visual field loss on health-related quality of life in glaucoma. the Los Angeles Latino Eye Study. Ophthalmology 2008; 115: 941-949.

6 Lisboa R, Chun YS, Zangwill LM, Weinreb RN, Rosen PN, Liebmann JM et al. Association between rates of binocular visual field loss and vision-related quality of life in patients with glaucoma. JAMA Ophthalmol 2013; 131(4): 486-494.

7 Murata H, Hirasawa H, Aoyama Y, Sugisaki K, Araie M, Mayama $\mathrm{C}$ et al. Identifying areas of the visual field important for quality of life in patients with glaucoma. PLoS One 2013; 8: e58695.

8 Cheng H-C, Guo C-Y, Chen M-J, Ko Y-C, Huang N, Liu CJ-1. Patient-reported vision-related quality of life differences between superior and inferior Hemifield visual field defects in primary open-angle glaucoma. JAMA Ophthalmol 2015; 133: 269-275.

9 Sukumar S, Spencer F, Fenerty C, Harper R, Henson D. The influence of socioeconomic and clinical factors upon the presenting visual field status of patients with glaucoma. Eye (Lond) 2009; 23: 1038-1044.

10 Fraser S, Bunce C, Wormald R, Brunner E. Socioeconomic status and late presentation of glaucoma. BMJ 2001; 322: 639-643.

11 Buys YM, Jin Y-P. Socioeconomic status as a risk factor for late presentation of glaucoma in Canada. Can J Ophthalmol 2013; 48: 83-87.

12 Hoevenaars JGMM, Schouten JSAG, van den Borne B, Beckers HJM, Webers CAB. Socioeconomic differences in glaucoma patients' knowledge, need for information and expectations of treatments. Acta Ophthalmol Scand 2006; 84: 84-91.

13 Nelson-Quigg JM, Cello K, Johnson CA. Predicting binocular visual field sensitivity from monocular visual field results. Invest Ophthalmol Vis Sci 2000; 41: 2212-2221.

14 Asaoka R, Crabb DP, Yamashita T, Russell RA, Wang YX, Garway-Heath DF. Patients have two eyes!: binocular versus better eye visual field indices. Invest Ophthalmol Vis Sci 2011; 52: 7007-7011.

15 Lin J-C, Chie W-C. Psychometric validation of the Taiwan Chinese version of the 25-Item National Eye Institute Visual Functioning Questionnaire. J Eval Clin Pract 2010; 16: 619-626.

16 Mangione CM, Lee PP, Gutierrez PR, Spritzer K, Berry S. Development of the 25-Item National Eye Institute Visual Function Questionnaire. Arch Ophthalmol 2001; 119: 1050-1058. 
17 Chatziralli IP, Sergentanis TN, Peponis VG, Papazisis LE, Moschos MM. Risk factors for poor vision-related quality of life among cataract patients. Evaluation of baseline data. Graefes Arch Clin Exp Ophthalmol 2013; 251: 783-789.

18 Globe DR, Varma R, Torres M, Wu J, Klein R, Azen SP. Selfreported comorbidities and visual function in a populationbased study: the Los Angeles Latino Eye Study. Arch Ophthalmol 2005; 123: 815-821.

19 Kong X, Zhu W, Hong J, Sun X. Is glaucoma comprehension associated with psychological disturbance and vision-related quality of life for patients with glaucoma? A cross-sectional study. BMJ Open 2014; 4: e004632.

20 Sun X, Zhang S, Wang N, Liang Y, Wang L, Fan S et al. Utility assessment among patients of primary angle closure/ glaucoma in China: a preliminary study. Br J Ophthalmol 2009; 93: 871-874.

21 Janz NK, Wren PA, Guire KE, Musch DC, Gillespie BW, Lichter PR. Fear of blindness in the Collaborative Initial Glaucoma Treatment Study: patterns and correlates over time. Ophthalmology 2007; 114: 2213-2220.
22 Zuo L, Zou H, Zhang J, Fei X, Xu X. Vision health-related quality of life in Chinese glaucoma patients. J Ophthalmol 2015; 2015: 1-9.

23 Luo R-J Liu S-R, Tian Z, Zhu W-H, Zhuo Y-H, Liao R-D. Rehabilitation of vision disorder and improved quality of life in patients with primary open angle glaucoma. Chin Med J (Engl) 2011; 124: 2687-2691.

24 Zhou C, Qian S, Wu P, Qiu C. Quality of life of glaucoma patients in China: sociodemographic, clinical, and psychological correlates - a cross-sectional study. Qual Life Res 2014; 23: 999-1008.

25 Ko Y-C, Hwang D-K, Chen W-T, Lee C-C, Liu CJ. Impact of socioeconomic status on the diagnosis of primary open-angle glaucoma and primary angle closure glaucoma: a nationwide population-based study in Taiwan. PLoS One 2016; 11(2): e0149698.

26 Ministry of Labor Republic of China (TAIWAN). International Labor Statistics. 10-3 Average effective age of retirement versus the official age, 2009-2014.

Supplementary Information accompanies this paper on Eye website (http://www.nature.com/eye) 